LA SERENA, veintiuno de febrero de mil novecientos noventa y cuatro.

\title{
VISTOS
}

A fojas 3 comparece don CARLOS OSORIO CORTES, constructor civil, domiciliado en calle Miguel Aguirre Perry, N 2025, La Serena, e interpone demanda contra la socieded LUIS MALUENDA SIERRA Y CIA. LIMITADA, representada por don LUIS MALUENDA SIERRA, ambos domiciliados en calle Guacolda N 30, Coquimbo, solicitando el pago de las prestaciones que señala, más intereses, reajustes y costos.

A fojas 8, evacuando el traslado la demandada, en mérito de los fundamentos que señala, solicita el rechazo de la demanda, con costas.

A fojas 9 vuelta se fijaron los puntos de prueba.

A fojas 11 a 24 rola prueba documental acompañada por la demandante.

A fojas 27 a 35 corre agrega prueba instrumental rendida por la demandada.

A fojas 40 se realiza la audiencia de conciliación y prueba, rindiendo la demandante la confesional con la declaración de LUIS MALUENDA SIERRA, y la testimonial con los dichos de don Jorge Yañez. Por su parte, la demandada rindió prueba confesional con los dichos del demandante, y testimonial con las declaraciones de JOSE ALONSO ROJAS ROJAS y DANIEL SEXTON.

A fojas 50 vuelta se citó a las partes a oir sentencia.

\section{O NSIDERANDO}

PRIMERO: Que a fojas 3 comparece don CARLOS OSORIO CORTES e interpone demanda en contra de LUIS MALUENDA SIERRA Y CIA. LTDA., representada por don Luis Maluenda Sierra, fundada en que fue contratado a contar del 1 de septiembre de 1993 con una renumeración de $\$ 600.000$, como administrador general de las obras desarrolladas por la empresa demandada como subcontratista en virtud del contrato de construcción de caminos y plataformas, llamado Proyecto Nevada, pactándose en la cláusula sexta del contrato que la duración de los servicios sería hasta la época de término de los trabajos, lo implicaba principalmente la construcción de 16.880 metros de camino y plataformas a ejecutar dentro de un plazo de 5 meses, contados desde su inicio del mes de septiembre y además la obligación de realizar la limpieza, mantención y reparación de los caminos hasta el 31 de marzo de 1993. Que el 14 de octubre se le puso término a su contrato aduciéndose la conclusión del trabajo, señalándose que el contrato de construcción contemplaba solamente 1600 metros lineales, no obstante que las obras para lo cual fue contratado continúan desarrollándose. 
Que en mérito de lo anterior, solicita se le cancelan las siguientes prestaciones: 1) Indemnización sustitutida del aviso previo $\$ 600.000 ; 2$ ) remuneraciones hasta la conclusión de los trabajos $\$ 3.000 .000$; y 3) gratificaciones año 1993 y fracción año tributario 1994, más intereses, reajustes y costas.

SEGUNDO: Que a fojas 8, evacuando el traslado la demandada, solicita su rechazo porque el trabajo para el cual fue contratado concluyó, firmándose el respectivo finiquito por las partes; que la cláusula sexta de su contrato debe ser interpretada correctamente en la parte que dice "hasta el término de los trabajos", ya que esta frase está de acuerdo a que los trabajos de construcción de caminos y otros proyectos Nevada no es la construcción de un camino cualquiera, sino que son diversos trabajos de manera que la sociedad demandada solamente ejecuta algunas obras con cierta clase de maquinarias y otros trabajos los ejecuta otra empresa con otras maquinarias; agrega que conforme al contrato de prestación de servicios entre Corma Ltda. y la empresa demandada, los trabajos de septiembre de 1993 hasta el 13 de octubre de 1993, era la construcción de 1620 metros lineales de caminos y para esta ejecución se necesitaban los servicios del demandante y desde esa fecha ya no existe en la empresa un administrador general pues no se le necesita. Que los trabajos son por partes o tramos y cada tramo necesita un determinado tipo de maquinarias y técnicos y que de ninguna manera puede entender que el contrato es para el proyecto Nevada pues esta mención se refiere al lugar donde se realizará el trabajo, pero no es parte en el proyecto donde funciona el establecimiento. Finalmente expone que no tiene derecho a gratificación porque no se ha pactado.

TERCERO: Que el actor para fundamentar su demanda acompañó la documental siguiente:

1.- Copia del contrato de construcción de caminos y plataformas.

2.- Constancia escrita de 11 de octubre de 1993 dirigida a la Inspección del Trabajo.

Llamó a absolver posiciones al representante de la demandada, don Luis Maluenda Sierra, quien reconoce que Corma Ltda. está a cargo de la construcción del proyecto Nevada y para ello celebra a su vez contratos con diversas empresas como subcontratistas, entre ellos su empresa, que los provee de equipo y personal, y que estos contratos son especiales porque dependen además de las condiciones climáticas del lugar y sus características geológicas, haciéndose los contratos por determinados tiempos y horas de acuerdo a sectores. Que el demandante fue contratado para llevar a cabo las obras ejecutadas en virtud del contrato de construcción de caminos y plataformas, pero referido al contrato que hizo CORMA con ellos; que efectivamente el contrato entre Corma y Cía. Minera Nevada consulta la construcción de 16.880 metros de caminos y platafor- 
mas; que su obra como subcontratista no fue ejecutar estos metros, sino que sólo se limitó a dar servicios diversos de maquinaria. perforistas y personal, existiendo también otro contratista para la construcción de ese camino; que el contrato del actor lo fue para administrar un tramo del camino durante el mes de septiembre y parte de octubre; que la cláusula sexta del contrato del actor dice asi porque se refiere a la parte general, ya que fue la labor que le pidieron; que la construcción de ese camino se realizó por su empresa, usando fundamentalmente compresores y aún se encuentran trabajando, porque los contratos son parcializados. Que su empresa no continúa trabajando allí; que el demandante mensualmente debía hacer 1.000 metros de camino porque era de 1500 metros el sector para el que los contrataron; que durante el mes de septiembre sólo hizo alrededor de 1600 metros y duró de 30 a 40 días; que él es el socio principal de la empresa demandada; que es socio de CORMA en un $45 \%$ y finalmente, dice que el contrato de prestación de servicios agregado a fojas 27 se refiere al contrato celebrado entre la empresa demandada y CORMA, que él no tiene ninguna relación con el contrato de construcción del camino agregado a fojas 11 , porque éste se refiere a especificaciones técnicas entre Compañía Minera Nevada y Corma, y que el contrato del actor se hizo a través de una empresa contratista para asegurarle una mayor estabilidad y por ese motivo el contrato que hizo Corma a Maluenda y Cía. se hizo solamente por esa cantidad de metros.

Rindió la testimonial don Jorge Yáñez, quien refiere haber trabajado con el demandante y que él lo hizo como administrador supervisor, para ver la parte personal, explosivos y otros, en las faenas mineras en construcción de caminos, pero si que la obra terminaba como en abril o mayo, que esto se lo dijo don Luis Maluenda; que en el lugar también había otros perforistas y los demás pertenecían a Nevada; que él trabajó hasta diciembre y aún seguían construyendo el camino y la plataforma, por lo que la empresa demandada siguió, lo que se notaba porque era el mismo personal y maquinaria; que luego que el demandante dejó de trabajar, siguió un Sr. Alonso. Preguntando para que diga en que punto se iniciaba y terminaba la construcción, responde que no se puede precisar porque son caminos en diversos sectores, en un cerro en que perforan a su alrededor, dependiendo de las vetas y muestras que sacan, y que la naturaleza entre un camino y el otro es el mismo, no variaba. Contrainterrogado dice que iba personal de $\mathrm{Ne}$ vada a medir los metros y hacían un listado de metraje por frente.

CUARTO: Que por su parte la demandada acompañó la siguiente documental:

1.- Contrato de prestación de servicios entre CORMA Ltda. y la empresa LUIS MALUENDA y $\mathrm{CIA}$;

2.- Copia del finiquito;

3.- Copia del contrato;

4.- Tres boletas de honorarios del demandante. 
Llamó a absolver posiciones al actor al tenor del pliego que rola a fojas 39 , quien reconoció haber firmado finiquito ante la Inspección del Trabajo y que corresponde al agregado en autos; que la empresa Corma y Luis Maluenda es el mismo; que la obra Nevada la desarrollaron tanto la empresa demandada como la de Luis Cortés, conjuntamente; que los subcontratistas deben atenerse a las instrucciones de los directivos de Nevada, quienes pueden hacer modificaciones dependiendo del lugar; que se le contrató para hacer el total del trabajo, 16.800 metros, el que estaba dividido por kilómetros y mensualmente debían hacerse un promedio de 3.200 metros; y diarios 104 metros, según lo exigía el contrato de Nevada; existía un programa de trabajo de acuerdo con la calidad del sector a trabajar; que el contrato para hacer los 16.800 metros lo conversó personalmente con don Luis Maluenda y el Gerente del proyecto, porque él se encontraba haciéndoles asesoría desde el mes de marzo del año pasado y trabajó agosto a honorarios y desde septiembre con contrato; que su labor la supervigilaba la Compañía Minera Nevada; que al término de sus labores habían 4.000 metros y algo más y él hacía un promedio del $75 \%$ de lo que se le exigía; que diariamente se hacían 104 metros; que el trabajo se hacía por sectores de acuerdo a las necesidades del terreno y la empresa y el 13 de octubre llevaban sobre los 4.000; que se le contrató por el total del proyecto, los 4.000 eran metros lineales, las plataformas se construían por metros cuadrados y el camino por metro lineal y a la fecha de su retiro estaba construída el $50 \%$ aproximado de plataforma, esto es, 400 metros cuadrados y finalmente reconoce que anteriormente le hizo trabajos al demandado y son suyas las boletas que se le exhiben agregadas a fojas 31,32 y 33 .

Rindió además la testimonial de José Rojas Rojas, quien afirma ser gerente general de Corma y Cía. Ltda., empresa que contrató los servicios de Luis Maluenda y Cía para ejecutar ciertos trabajos en el proyecto Nevada y el actor trabajó para esta última empresa como supervisor para la construcción de 1600 metros lineales de camino que tenía Luis Maluenda y Cía contratados con Corma, no renovándosele el contrato al actor porque era constructor civil y se necesitaba un profesional minero; por lo tanto no se le renovó el contrato a la empresa demandada respecto de la construcción porque ya había cumplido la etapa de los 1600 metros posteriormente la construcción desarrollándose con Luis Maluenda Sierra, ya que éste participa también en otra empresa Luis Maluenda e Hijos y además es socio de Corma, por lo que esta última contrata a cualquiera de estas empresas para hacer los trabajos, porque una es minería, la otra de movimiento de tierra y la otra de personal y estas empresas se subcontratan a su vez; agrega que los 1600 metros se terminaron en octubre, 700 el primer mes y 900 el mes de octubre, datos que quedan registrados en la productividad del informe diario y Nevada fiscaliza y registra el cumplimiento de las etapas, pero Luis Maluenda y Cía. nunca informó los índices de productividad diaria y fue uno de los motivos principales que no se le renovó el contrato a la empresa demandada. Contrainterrogado acerca de si al cese de los servicios del actor se habían construído por otros subcontratistas más de 3000 metros de camino, además de la empresa demandada, responde que técnicamente no se puede y los únicos 
metros lineales construídos fueron hechos por Luis Maluenda y Cía. y que en poder de Nevada debería existir un libro que registrara lo anterior, pero el libro no registra estas anotaciones; contrainterrogado acerca de si el demandante fue cesado en su trabajo por falta de idoneidad personal, responsable que fue vetado por Compañía Minera Nevada, por problemas técnicos solamente, porque lo ideal era un minero y éste era constructor civil. Y de Daniel Saxton quien señala que el actor dejó de trabajar por la conclusión del contrato entre Compañía Minera Nevada y la empresa demandada; que el contrato entre Corma y Luis Maluenda y Cía, era por 1600 metros aproximados y terminó el día 11 de octubre más o menos y el actor fue contratado para administrar la parte de ese contrato y no sabe si hubo algún problema con el demandante; que él es administrativo y contratado por Luis Maluenda y Cía., empresa que funciona en un mismo lugar con las demás empresas de don Luis Maluenda: Luis Maluenda Sierra, Luis Maluenda e Hijos y CORMA ubicadas en Guacolda $\mathrm{N}^{2} 30$, de Coquimbo, y en sus labores administrativas también hace algunos trabajos para esas empresas y la cantidad de metros construídos consta en el estado de pago final que llegó a la empresa. Repreguntado dice que en la obra existen inspectores de la empresa Nevada y si una persona no cumple, pide que la cambien y en el caso del demandante estimaron que debía desempeñar estas labores un ingeniero civil en minas y que era muy difícil que el actor siguiera por la naturaleza de las labores.

QUINTO: Que la discusión en estos autos se centra en determinar si a la fecha en que el actor fue despedido, 14 de octubre, habían concluído los trabajos para los que fue contratado.

Según la demandante, de acuerdo a las cláusulas 1 y 6 del contrato, fue contratado por la construcción de 16.880 metros de caminos de exploración, de acuerdo al contrato suscrito entre Cía. Minera Nevada y la empresa Corma., la que a su vez contrató al actor.

Por su parte la demandada sostiene que el actor fue contratado solamente para la construcción de 1620 metros lineales de acuerdo a un subcontrato celebrado entre Corma y Luis Maluenda y Cía., que se encuentra agregado a fojas 27 , construcción que concluyó a la fecha en que se puso término a sus servicios, el 14 de octubre de 1993.

SEXTO: Que según el documento " contrato de construcción de caminos y plataformas Compañía Minera Nevada S.A." de fecha 23 de agosto de 1993, esta última encargó a

CORMA la construcción de 16.800 metros aproximados de caminos y plataforma del Proyecto de Nevada y otras obras, contrato que tiene un plazo de cinco meses a partir del 1 de septiembre de 1993, según cláusula cuarta, comtemplando en el punto 5.3 la obligación de Corma de designar un supervisor como autoridad para atenderse con la empresa; y en el punto 11.1 se expresa que Nevada está expresamente facultada para 
solicitar a Corma por escrito y a través del supervisor, el retiro de los trabajadores que no cumplan con las condiciones de conocimiento o experiencia profesional.

SEPTIMO: Que consta asimismo del documento acompañado por la demandada a fojas 27 -contrato de prestación de maquinaria entre las empresas CORMA y Luis Maluenda y Compañía para la ejecución del contrato entre la Compañía Minera Nevada y Corma, cláusula tercera-, que Corma no cuenta con personal ni maquinarias para ejecutar el contrato celebrado, referido en el considerando anterior, "para cuyo efecto se ha enviado personal suficiente para preparar la ejecución de los tramos exigidos por el proyecto Nevada, que consiste en la construcción de mil seiscientos veinte metros (1620) lineales", señalándose, además, en su cláusula séptima que el contrato "empezará con fecha primero de septiembre de mil novecientos noventa y tres y tendrá una duración estimada de treinta días corridos, sin perjuicio que por la cacterísticas del terreno en cuestiones climáticas, este período se alargue por un lapso no superior a diez días".

OCTAVO: Que analizadas las pruebas rendidas de conformidad con las normas de la sana crítica, esta sentencia concluye que la causal no se encuentre acreditada, en base a los siguientes fundamentos.

a) La labor para la que fue contratado el actor es amplia, según reza la cláusula primera de su contrato, y que señala "el trabajador se compromete a ejecutar el trabajo de administradora general del contrato en el establecimiento de Construcción de Caminos y Plataformas denominado Proyecto Nevada", contrato que durará "hasta el término de los trabajos", situación que coincide plenamente con el contrato celebrado entre la Compañía Minera Nevada y Corma en que se señala como objetivo la construcción de 16.880 metros aproximados de caminos y otras obras.

b) Que el contrato se prestación de servicios de fojas 27 celebrado entre Corma y la empresa demandada, no estipula derechamente que lo sea para la construcción de 1620 metros lineales, sino que refiere la cláusula tercera que se ha enviado personal suficiente para preparar la ejecución de los tramos exigidos por el proyecto Nevada, que consiste en la construcción de 1620 metros lineales, por lo que podría ser que exista otro subcontrato por otro período.

c) Que tal como lo reconoce el propio representante de la demandada y lo confirman los testigos de su parte, don Luis Maluenda Sierra también es socio de la empresa CORMA con quien subcontrata, además de ser socio de las otras empresas subcontratistas en dichas labores, señalando el testigo Sexton que todas las empresas funcionan en un mismo lugar y que él como administrativo, también hace trabajos para estas empresas. 
d) Que los propios testigos de la demandada declararon que la causal de término del subcontrato lo fue porque Compañía Minera Nevada exigía que el supervisor fuera un Ingeniero Civil en Minas y no Constructor Civil como era el actor;

e) Que aún en el caso que fuera efectivo que el subcontrato - origen del contrato actor - lo hubiere sido para la construcción de 1620 metros lineales, en virtud de la cláusula no había estado acreditada, toda vez que señala un plazo de treinta días corridos y un alargue por un lapso no superior a diez días a contar del primero de septiembre, lo que arroja un plazo hasta el 10 de octubre y el actor fue despedido el 14 de octubre de 1993; y

f) Finalmente esta sentenciadora tuvo presente el hecho que el actor, de acuerdo a su confesión, reconoce que participó en el proyecto desde el mes de marzo y trabajó en el mismo a honorarios en los meses anteriores a su contrato, por lo que estaba en antecedentes de cual era el objeto de su contrato.

NOVENO: Que atendido entonces a lo concluídoprecedentemente, se dará lugar a la demanda en cuanto pide la indemnización sustitutiva del aviso previo y el pago de las renumeraciones hasta el término de su contrato; esto es cinco meses a contar del 1 de septiembre de 1993.

DECIMO: Que se rechaza el pago de las gratificaciones atendido el mérito del finiquito agregado a fojas 2 , en que aparece que solamente se reservó el derecho a cobrar remuneración por dos días, y el reclamo de la causal de despido.

Por estos fundamentos y atendido lo dispuesto por los artículos 1, 3, 7, 62, 429, 430, 432, del código del Trabajo; y artículos 10 y 15 de la Ley № 18.010; SE DECLARA:

QUE HA LUGAR a la demanda de fojas 3 sólo en cuanto la demandada deberá pagar al actor la suma de $\$ 600.000$ por indenmización sustitutiva del aviso previo y $\$$ 2.120 .000 por remuneración.

Que no se condena en costas a la demandada por no haber sido totalmente vencida.

Que las sumas ordenadas pagar precedentemente, deberán hacerse efectivas de conformidad con lo dispuesto por los artículos 62 del Código del Trabajo, en relación con el $\mathrm{N}^{\mathrm{a}} 15$ de la Ley 19.010 .

REGISTRESE Y NOTIFIQUESE. 
Dictada por doña MARTA MALDONADO NAVARRO, Juez Letrado titular de este Primer Juzgado del Trabajo. Autoriza doña NORMA RIVERA ARAYA; Secretaria titular.

La Serena, tres de Mayo de mil novecientos noventa y cuatro.

\section{Vistos}

Se reproduce la sentencia en alzada y se tiene, además, presente:

Que al actor se le contrató para administrar "las obras" pero no se especificó de cuales obras se trataba, aclaración que era necesaria toda vez que la empresa empleadora "Luis Maluenda Sierra y Cía Ltda." tenía diversos trabajos que realizar.

Que es inconcuso que correspondía al demandado o sea a la empresa empleadora acreditar la licitud del despido, esto es que se había dado la condición que permitía a éste poner término al contrato del empleado, o sea en este caso, que la labor estaba concluída cosa que no hizo ya que era previo para ello haber probado, que labor precisa correspondía realizar al demandante.

Y visto, lo dispuesto en los artículos 436 del Código del Trabajo y demás disposiciones citadas, se confirma la sentencia apelada de veintiuno de Febrero último, escrita a fojas 51 .

Cada parte pagará sus costas.

Regístrese y devuélvase.

Redacción del Sr. Ministro Titular, don José Pavisic Dragnic.

Rol No 783 - T.-

Santiago, veintitrés de enero de mil novecientos noventa y cinco

\section{Vistos}

Atendido el mérito de los antecedentes, lo informado por los jueces recurridos y por no existir falta ni abuso susceptible de enmendarse por esta vía, se declara SIN LUGAR el recurso de queja interpuesto en lo principal de fojas 2, con costas.

Se aplica a beneficios de la Corporación Administrativa del Poder Judicial la consignación a fojas 5 Gírese cheque.

Se impone, además, al recurrente una multa, con el mismo destino, de dos unidades tributarias mensuales, suma que deberá depositar en la cuenta corriente del Tribunal dentro de quinto día, bajo aprecibimiento legal. El Sr. Secretario constará el cumplimiento de dicha obligación.

Regístrese, comuníquese y archívese. 
Proveído por la Excma. Corte Suprema. Presidente Sr. Zurita, Ministros Sres. Dávila, Araya, abogado integrante Sr. Montes.

Queja del Trabajo №3.936

COMENTARIO A UN FALLO DEL PRIMER JUZGADO DE LETRAS DEL TRABAJO DE LA SERENA, CONFIRMADO EN SEGUNDA INSTANCIA Y DICTADO SIN FALTA O ABUSO POR DECLARACIÓN DE LA EXCMA. CORTE SUPREMA.

El artículo 159 № 5 del Código del Trabajo previene que el contrato de trabajo terminará por conclusión del trabajo o servicio que dio origen al contrato.

De la norma preinserta es dable concluir que constituye causa leítima de terminación de un contrato de trabajo el hecho de haber concluido el trabajo o servicio que le dio origen, con tal, a nuestro juicio, que ese trabajo o servicio haya sido la causa originaria o generadora del contrato, y que esté claramente definida en un grado de precisión tal que pueda comprobarse objetivamente el monento de su concurrencia.

Esta norma debe entenderse en íntima relación con lo previsto en el artículo 10 № 3 del mismo cuerpo legal, en orden a que el contrato de trabajo debe contener entre otras, la estipulación acerca de la naturaleza de los servicios que debe prestar el trabajador y al № 7 del mismo precepto, que se refiere a estipulaciones generales de los contratantes, entre las que debe señalarse la condición o evento de que el contrato concluirá al término de una definida parte o totalidad de una faena, obra, trabajo o servicio.

Invocada la causal por el empleador, corresponde a él soportar el peso de la prueba de su justificación o efectividad.

En el fallo en comento se ha establecido que el demandante prestó servicios para una empresa subcontratísta de otra, que, a la vez, era contratista de una tercera.

La naturaleza del servicio del actor era administrar una obra de construcción de caminos que ejecutada su empleador. La determinación de los servicios es amplia y definida a una función de carácter permanente, cual es la administración de las obras de construcción antedichas.

Aunque no existe inconveniente en contratar a un trabajador para la realización de un trabajo o servicio que corresponda a una parte o fase de una obra mayor, tal circunstancia debe estar claramente especificada en el contrato de una manera capaz de ser constatada objetivamente, a fin de no dejar al trabajador en la indefensión ante una eventual invocación extemporánea o indebida de la causal de despido.

Tal es el caso en análisis, en que no se precisó que el trabajador fuere contratado para la administración de una determinada fase de la obra mayor, habida consideración 
que si bien es cierto el empleador estaba ejecutando una parte del total de los caminos programados por así haberlo acordado con su "mandante", el contrato de construcción se refería a toda la obra mayor.

Analizadas las pruebas y antecedentes del juicio conforme a las reglas de la sana crítica, resulta convincente a juicio del sentenciador que el actor fue contratado en calidad de administrador para toda la obra de construcción de caminos, aún cuando ésta se hubiere estado desarrollando en una determinada longitud.

Resulta interesante la falta de desarrollo por parte del demandante de la consideración establecida en el inciso final del artículo $3^{2}$ del Código del Trabajo, en orden a que para los efectos de la legislación laboral y de seguridad social, se entiende por empresa toda organización de medios personales, materiales e inmateriales, ordenados bajo una misma dirección, para el logro de fines económicos, sociales, culturales o benéficos, dotada de una individualidad legal determinada.

Aparentemente el demandado y su "mandante" pudieron ser calificados como una misma empresa, desde el momento que la dirección estaba ejercida por una misma persona, que se trataba de una misma organización de recursos, que su composición pátrimonal o societaria era más o menos similar, etc., que junto a la concurrencia de los requisitos legales, habría reforzado aún más la posición jurídica del actor.

\author{
Mario Carvallo Vallejos \\ Abogado, Prof. Escuela de Derecho \\ U.C.N. Coquimbo
}

